Vol.01/ No. 02

Pages: 68-76

https://www.irojournals.com/itdw/

DOI: https://doi.org/10.36548/jitdw.2019.2.002

\title{
INTELLIGENT TRAFFIC CONTROL DEVICE MODEL USING AD HOC NETWORK
}

\author{
Dr. Robert Bestak \\ Department of Tele Communication Engineering, \\ Czech Technical University in Prague, \\ Prague, Czech Republic. \\ Email: robert.bestak@,fel.cvut.cz
}

\begin{abstract}
The advancements in the technologies related to the wireless communication systems has made the vehicular adhoc networks prominent area of research in the automobile industry. The absolute volume of road traffic affects the safety, convenience and the efficiency of the traffic flow in the urban areas. So the paper scopes in developing an intelligent traffic control device model using the adhoc network to ameliorate the traffic flow. The proposed system enhances the convenience in travel by gathering the information of the vehicles along with the density of the vehicles and the movement of the vehicles on road. The device is modelled using the MATLAB and examined over the traffic flow on the peak hours as well as the normal hours and the holidays to understand its intelligent traffic control. The results obtained shows that the performance improvement in optimizing the traffic congestion through the proposed method is better compared to the existing methodologies used in traffic controlling.
\end{abstract}

Keywords: Intelligent Traffic control, Adhoc networks, wireless sensor networks,

\section{INTRODUCTION}

The necessity for developing an intelligent traffic control device has become very essential nowadays as the density of the vehicles are increasing every year at an alarming rate in the most of the developing countries. The increasing traffic congestion as a result of increasing number of vehicles affects the safety, convenience and the patience of the travelers. Even the latest technological developments improving the existing traffic and the road infrastructure becomes insufficient on certain situations where the waiting time is unavoidable, such VIP Visits, Crossing of emergency vehicles and sudden road damages.

The current technology aids the traffic condition utilizing the wired sensor, inductive loops and the surveillance cameras etc. to observe the position as well as the speed of the vehicles, these methods were found to be too costly 
Journal of Information Technology and Digital World (2019)

Vol.01/ No. 02

Pages: 68-76

https://www.irojournals.com/itdw/

DOI: https://doi.org/10.36548/jitdw.2019.2.002

in implementing and maintaining. More over the conceptual system that enables the interaction between the vehicles are also not practical in countries like India as there are more number of vehicles in basic model and people unaware and hesitating to adopt to such kind of systems. The accuracy of the devices were are also clubbed with the environmental conditions and it was hard for the traffic light control device to adapt to the continuously changing traffic density and the congestion.

So to handle the application that are critical and the situations in real time, the wireless sensor networks that are specific type of a wireless adhoc network communicating using the network framed with the sensors is put forth as a solution. The wireless sensor networks is well suited to handle the traffic flow in time of congestion and emergencies and are highly preferred due to its robustness, flexibility and limited power requirements. So the proposed system uses the wireless sensor network and develops a traffic control device model, gathering the speed and the location of the vehicles and tries to minimize the average waiting time of the vehicles during the peak hours and optimizes the traffic flow utilizing the free roads. The paper utilizes the sensors and the microcontrollers to perfectly regulate the traffic flow, the rest of the paper is organized with the related works providing the ground work of the proposed traffic control device, in the section 2, the proposed intelligent traffic control in section 3 , performance analysis in section 4 and the summarization as conclusion in section 5.

\section{RELATED WORKS}

Raj, Jennifer S., et al [1] proposes the wireless sensor for the transfer of information, optimizing the quality of service metrics and the power consumption applying the clustering based fuzzy rule system with the short route determination method. Smys, S. et al [2] provides the advantage of employing the wireless sensor networks in the big data applications. The author proposes an algorithm to select the nodes with the substantial resources to have an energy aware secure information conveyance. Pandian, M. Durai et al [3] the author proposes the wireless network selection and handover in the heterogeneous networks using the grey relationship analysis and the particle warm optimization.

The authors Smys, S., and Jennifer S. Raj et al [4] proposes the "stochastic data traffic model to ameliorate safety and comfort for driving by overcoming the problems of traffic congestion, interference and jamming. It also addresses the handover (HO) issuethat occurs during fast mobility Along with the quality parameters of the system such as throughput, packet delivery ratio and delay" 
Journal of Information Technology and Digital World (2019)

Vol.01/ No. 02

Pages: 68-76

https://www.irojournals.com/itdw/

DOI: https://doi.org/10.36548/jitdw.2019.2.002

The author Duraipandian, $\mathrm{M}$ et al [5] proposed the mobile adhoc networks based on the machine learning techniques to, "exploiting the node information's to establish a route that is short and stable in order to minimize the energy consumption, transmission delay, and improve the delivery ratio of the packets, enhancing the throughput".

The author Sathesh et al [6] put for the "the hybridized optimization technique to handle the multi-objective problem faced by these decentralized networks and achieves the throughput, energy consumption and the network longevity. The author Manoharan, S.et al [7] in his paper proposes the "Measures to improve the probability of the safety is put forward in the paper for the cars with the capability of the self-driving built with the artificial intelligence enabled processors"

Haoxiang, and Smys et al [8] "puts forth the modified Ant colony optimization that integrates the clustering technique to frame optimal shortest route for the internet access in the vehicular network to manage its mobility and the topology changes. The process of optimal route selection taking into consideration the mobility and the dynamic topology in of the proposed method improves the grounds of bandwidth utilization throughput delay and link life expectancy"

Koresh, Mr H. James Deva et al [9] proposes the utilization of the computer vision in traffic sign sensing offering a high reliability and spatial variance. Jyothirmai et al 10] proposes the "Secured self-organizing network architecture in wireless personal networks." Anguraj, Dinesh Kumar, and S. Smys et al [11] put forth the "'Trust-based intrusion detection and clustering approach for wireless body area networks." Praveena, A., and S. Smys et al [12] proposes the a survey on the issues of data privacy in social network sites based on the Anonymization in social networks" Smys, S., G. Josemin Bala, and S. Jennifer et al [13] proposes the "Mobility management in wireless networks using power aware routing."

Anda, Joey et al [13] puts forth the "VGrid: vehicular adhoc networking and computing grid for intelligent traffic control." The figure.1 below provides the architecture of the V GRID. 
Journal of Information Technology and Digital World (2019)

Vol.01/ No. 02

Pages: 68-76

https://www.irojournals.com/itdw/

DOI: https://doi.org/10.36548/jitdw.2019.2.002

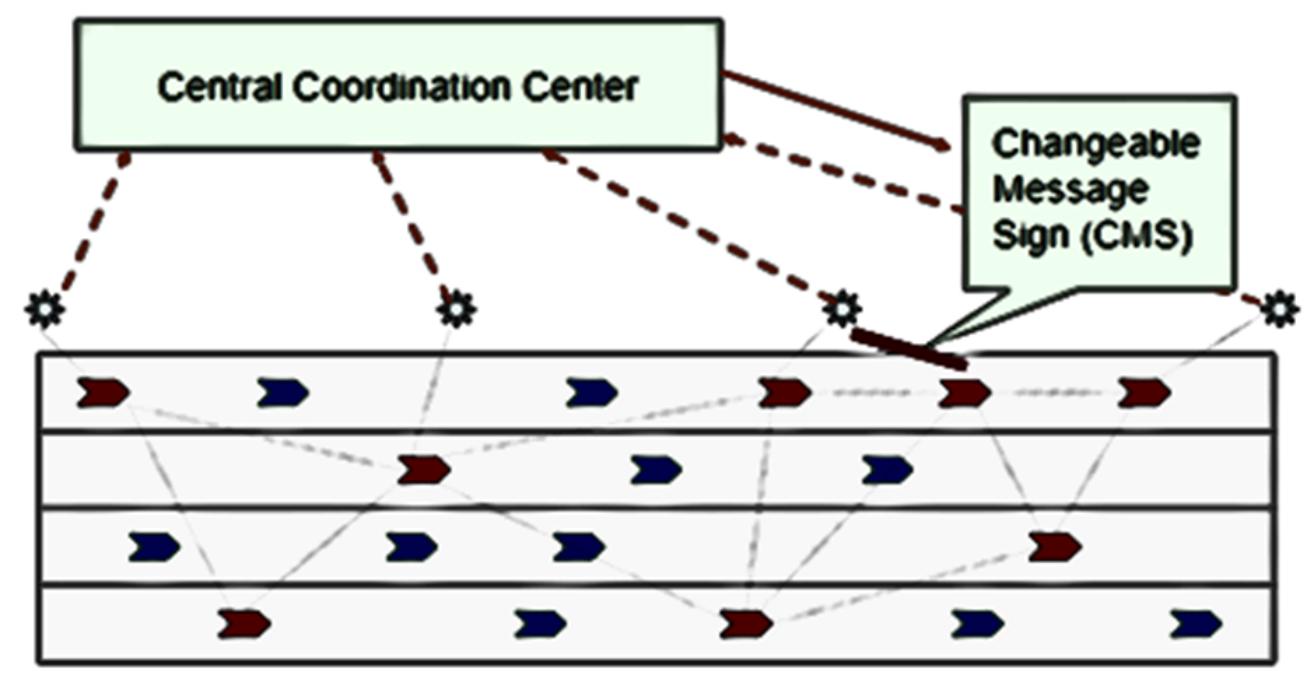

Figure.1 V-GRID Architecture

\section{PROPOSED INTELLIGENT TRAFFIC CONTROL}

The proposed intelligent traffic flow controlling device is modelled using sensor networks and the microcontroller to process the information, gathered and regulate the traffic flow, the sensors, locating the vehicles and the speed of the vehicles are placed in the every lamppost that is situated in the distance of the 30 kilometers, the information gathered are transmitted wireless to the microcontroller that does a simultaneous processing to provide the solutions required. The figure. 2 below shows the simple diagram of the proposed intelligent traffic control.

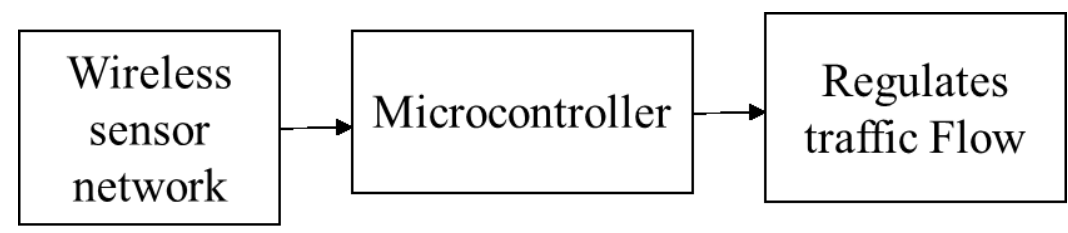

Figure.2 Simple -Proposed Flow Diagram 
Journal of Information Technology and Digital World (2019)

Vol.01/ No. 02

Pages: 68-76

https://www.irojournals.com/itdw/

DOI: https://doi.org/10.36548/jitdw.2019.2.002

The intelligent traffic control utilizes the TMOTESKY-MTMCM3000-MSP to monitor and collect the particular of the vehicles, the information noted down are also includes the number of the vehicles and the types of the vehicles, are sensed as the any type of sensors can be integrated and used along with TMOTESKY, it is featured with the chipcon CC2420 radio for wireless communication and leverages standards like USB and the IEEE802.154 to operate seamlessly with the other devices. The CC2420 is highly configurable with default radio settings, the proposed model uses the Zig Bee to extend communication from sensors to the controller.

Once the information of the vehicles are transmitted to the controller the controller is programmed to detect the sequenced numbered from 0 to 3 clock wise, start initiating the red state to all the roads, assuming any one of the road to begin the cycle starts at the $4^{\text {th }}$ cycle where the cycle length is determined using the following equation (1) given below

$$
\text { Cycle }_{\text {length }}=\frac{\frac{1}{2}-\text { time }}{\text { lost }}
$$

Where, thedeg $g_{\text {saturation }}=\sum_{i=1}^{m} L_{i}$, where the $\mathrm{m}$ is the phases in the traffic lights and the, $\mathrm{L}$ is the saturate flow. The time for every vehicle sensed is determined and the traffic flow is regulated estimating the volume of the vehicles expressed as the equivalent vehicles per time. At every cycle any two roads opposite to each other are completely regulated, making the vehicles on all the lanes to wait only for 3 cycles, following the round robin schedule the equal time slot is allotted to ever road.

\subsection{WORKING OF THE PROPOSED ALGORITHM}

The sensor transmit the particulars of the vehicles regularly to the controller for every 60 seconds and he controller on receiving the particulars analyses the speed of the vehicles estimates the location and identifies the type of the vehicle and sets priorities to the lane and initiates green signal to the lane with the emergency signal until the emergency vehicle has crossed and turns on the red state initiating the green signal to the lane with the next priority. If the lane in the middle is initiated with the green signal either the right lane is also initiated with the green or the opposite lane is initiated with the green, similar smart decision is initiated in the signals according to the traffic density. 
Journal of Information Technology and Digital World (2019)

Vol.01/ No. 02

Pages: 68-76

https://www.irojournals.com/itdw/

DOI: https://doi.org/10.36548/jitdw.2019.2.002

In countries like India the roads are segregated into four tracks according to the speed level of the vehicles, the track that comes in the left most corner is for vehicles with the low speed and the track that is in the right most corner is for the vehicles with high speed and other two are for vehicles with the moderate speed, the sensors fixed senses the track change of vehicles according to their speed and raises alarms until the vehicle is in its proper track. The right corner track is usually allotted for the emergency vehicles and the vehicles that take a right turn. The figure. 3 below shows the effective traffic management imitated by the intelligent traffic control device.

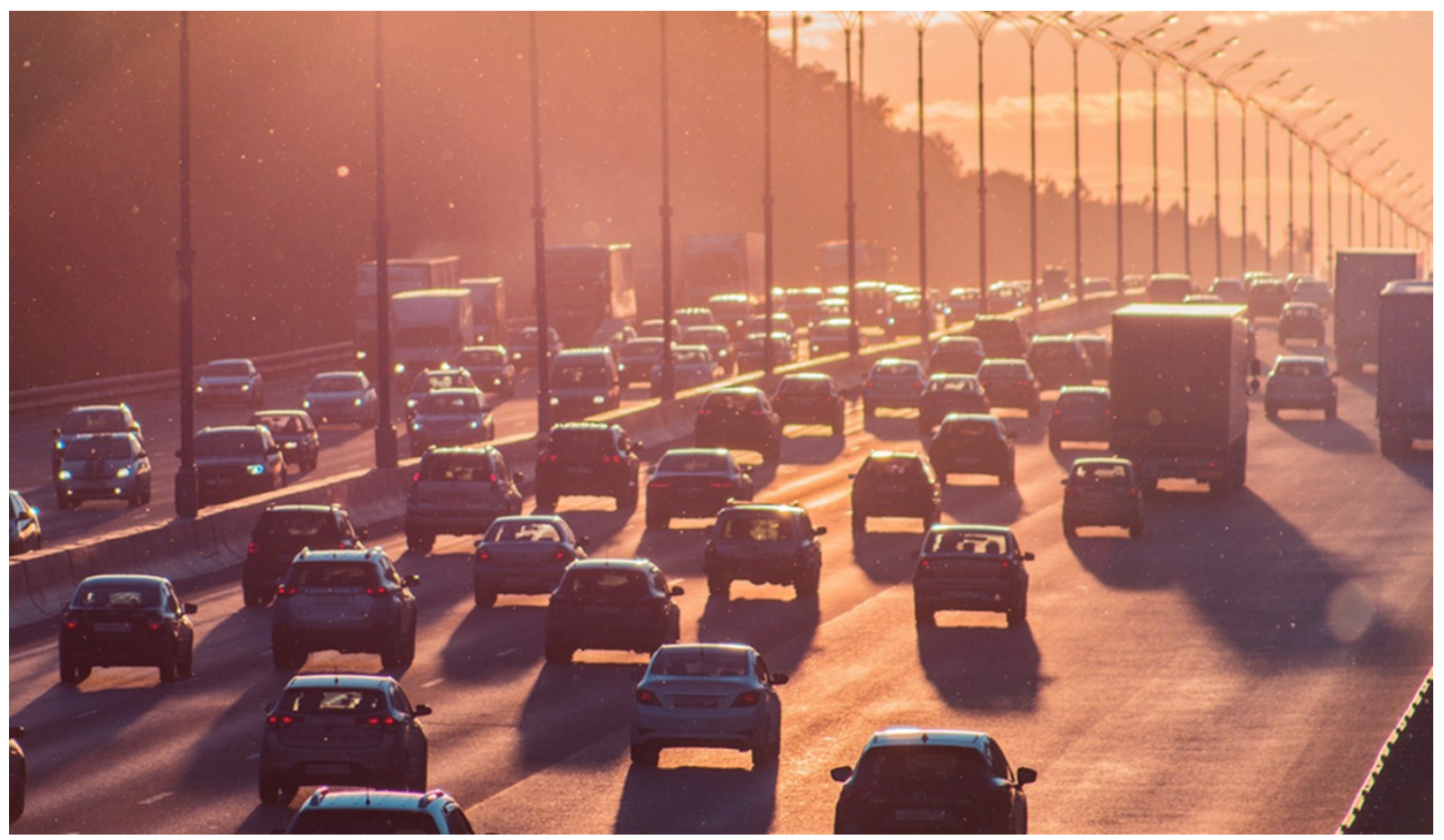

Figure. 3 Intelligent Traffic Control

\section{RESULTS}

The proposed model was designed using the MATLAB and was tested in the peak hours and the normal hours of a day and as well as on the holidays the, the results were observed on the basis of the distance, speed, vehicle volumes, cycle length and the delay second experienced by every vehicles. The table.1 below provides the result observed under different scenarios 
Journal of Information Technology and Digital World (2019)

Vol.01/ No. 02

Pages: 68-76

https://www.irojournals.com/itdw/

DOI: https://doi.org/10.36548/jitdw.2019.2.002

\begin{tabular}{|c|c|c|c|c|c|c|}
\hline \multirow{2}{*}{ Parameters } & \multicolumn{2}{|c|}{ Peak Hours } & \multicolumn{2}{c|}{ Normal Hours } & \multicolumn{2}{c|}{ Holidays } \\
\cline { 2 - 7 } & Traditional & Proposed & Traditional & Proposed & Traditional & Proposed \\
\hline Distance (m) & 380 & 380 & 290 & 290 & 200 & 200 \\
\hline Avg. speed (Km/h) & 60 & 60 & 80 & 80 & 100 & 100 \\
\hline Avg. Waiting Time(s) & 480 & 60 & 320 & 40 & 120 & 30 \\
\hline Vehicle Volume (V/h) & 3300 & 3300 & 1000 & 1000 & 400 & 400 \\
\hline Cycle length (s) & 72 & 72 & 35 & 35 & 20 & 20 \\
\hline Stop Number & 10 & 5 & 6 & 2 & 4 & - \\
\hline
\end{tabular}

Table.1 Results Observed

The results observed shows that the proposed method effectively controls the traffic reducing the average waiting time of the vehicles that is cause by the traditional traffic rules, this result obtained for the proposed system works with same efficiency for the peak hours as well as the holidays and the normal hours. The waiting period of the vehicles are reduced to a $45 \%$ compared to the conventional traffic policy. 
Journal of Information Technology and Digital World (2019)

Vol.01/ No. 02

Pages: 68-76

https://www.irojournals.com/itdw/

DOI: https://doi.org/10.36548/jitdw.2019.2.002

\section{CONCLUSION}

The proposed intelligent traffic control device utilizes the wireless sensor networks and the microcontrollers to effectively regulate the traffic and reduce the average waiting time of the vehicles. By gathering the particulars of the vehicles and regulating the flow according to the exigency of the vehicles. The result obtained shows the considerable reduction achieved in the average waiting time of the vehicles. In future the paper plans to extend the work monitoring the $\mathrm{Co} 2$ emission of the vehicle's and engaging a context aware traffic flow applying the machine learning algorithms.

\section{References}

[1] Raj, Jennifer S., and A. Basar. "QoS optimization of energy efficient routing in IoT wireless sensor networks." Journal of ISMAC 1, no. 01 (2019): 12-23.

[2] Smys, S. "ENERGY-AWARE SECURITY ROUTING PROTOCOL FOR WSN IN BIG-DATA APPLICATIONS." Journal of ISMAC 1, no. 01 (2019): 38-55.

[3] Pandian, M. Durai. "ENHANCED NETWORK SELECTION AND HANDOVER SCHEMA FOR HETEROGENEOUS WIRELESS NETWORKS." Journal of ISMAC 1, no. 03 (2019): 160-171.

[4] Smys, S., and Jennifer S. Raj. "A STOCHASTIC MOBILE DATA TRAFFIC MODEL FOR VEHICULAR AD HOC NETWORKS." Journal of Ubiquitous Computing and Communication Technologies (UCCT) 1, no. 01 (2019): 55-63.

[5] Duraipandian, M. "PERFORMANCE EVALUATION OF ROUTING ALGORITHM FOR MANET BASED ON THE MACHINE LEARNING TECHNIQUES." Journal of trends in Computer Science and Smart technology (TCSST) 1, no. 01 (2019): 25-38.

[6] Sathesh, A. "OPTIMIZED MULTI-OBJECTIVE ROUTING FOR WIRELESS COMMUNICATION WITH LOAD BALANCING." Journal of trends in Computer Science and Smart technology (TCSST) 1, no. 02 (2019): 106-120.

[7] Manoharan, S. (2019). AN IMPROVED SAFETY ALGORITHM FOR ARTIFICIAL INTELLIGENCE ENABLED PROCESSORS IN SELF DRIVING CARS. Journal of Artificial Intelligence, 1(02), 95-104.

[8] Haoxiang, Wang, and S. Smys. "QOS ENHANCED ROUTING PROTOCOLS FOR VEHICULAR NETWORK USING SOFT COMPUTING TECHNIQUE." Journal of Soft Computing Paradigm (JSCP) 1, no. 02 (2019): 91-102. 
Journal of Information Technology and Digital World (2019)

Vol.01/ No. 02

Pages: 68-76

https://www.irojournals.com/itdw/

DOI: https://doi.org/10.36548/jitdw.2019.2.002

[9] Koresh, Mr H. James Deva. "COMPUTER VISION BASED TRAFFIC SIGN SENSING FOR SMART TRANSPORT." Journal of Innovative Image Processing (JIIP) 1, no. 01 (2019): 11-19.

[10] Jyothirmai, Pondi, Jennifer S. Raj, and S. Smys. "Secured self-organizing network architecture in wireless personal networks." Wireless Personal Communications 96, no. 4 (2017): 5603-5620

[11] Anguraj, Dinesh Kumar, and S. Smys. "Trust-based intrusion detection and clustering approach for wireless body area networks." Wireless Personal Communications 104, no. 1 (2019): 1-20

[12] Praveena, A., and S. Smys. "Anonymization in social networks: a survey on the issues of data privacy in social network sites." Journal of International Journal Of Engineering And Computer Science 5, no. 3 (2016): 15912-15918.

[13] Smys, S., G. Josemin Bala, and S. Jennifer. "Mobility management in wireless networks using power aware routing." In 2010 International Conference on Intelligent and Advanced Systems, pp. 1-5. IEEE, 2010.

[14] Anda, Joey, Jason LeBrun, Dipak Ghosal, Chen-Nee Chuah, and Michael Zhang. "VGrid: vehicular adhoc networking and computing grid for intelligent traffic control." In 2005 IEEE 61st Vehicular Technology Conference, vol. 5, pp. 2905-2909. IEEE, 2005. 